\title{
Botulinum Toxin in Neurogenic Detrusor Overactivity
}

\author{
Carlos Arturo Levi D’Ancona, Rúiter Silva Ferreira, Mauricio Carneiro Rassi \\ Division of Urology, University of Campinas School of Medicine, Campinas, Sao Paulo, Brazil
}

\begin{abstract}
Purpose: To evaluate the effects of botulinum toxin on urodynamic parameters and quality of life in patients with neurogenic detrusor overactivity.

Methods: Thirty four adult patients with spinal cord injury and detrusor overactivity were selected. The patients received 300 units of botulinum toxin type A. The endpoints evaluated with the episodes of urinary incontinence and measured the maximum cystometric capacity, maximum amplitude of detrusor pressure and bladder compliance at the beginning and end of the study ( 24 weeks) and evaluated the quality of life by applying the Qualiveen questionnaire.

Results: A significant decrease in the episodes of urinary incontinence was observed. All urodynamic parameters presented a significant improvement. The same was observed in the quality of life index and the specific impact of urinary problems scores from the Qualiveen questionnaire. Six patients did not complete the study, two due to incomplete follow-up, and four violated protocol and were excluded from the analyses. No systemic adverse events of botulinum toxin type A were reported.

Conclusions: A botulinum toxin type A showed a significantly improved response in urodynamics parameters and specific and general quality of life.
\end{abstract}

Keywords: Overactive urinary bladder; Botulinum toxins; Quality of life; Urodynamics

\section{INTRODUCTION}

The major goal of treatment of patients with neurogenic detrusor dysfunction is the preservation of renal function, promotion of urinary continence, avoidance of urinary tract infection, and preservation of quality of life (QoL) [1]. These patients frequently struggle with urinary incontinence that may severely affect their QoL [2]. Spinal cord injury (SCI), one of the most frequent causes of neurogenic detrusor dysfunction in young adults, is often associated with neurogenic detrusor overactivity (NDO).

The introduction of clean intermittent catheterization and the use of antimuscarinic drugs present important tools in the treatment of NDO. These treatments increase urinary continence, decrease urinary tract infections, and preserve renal function [3-5]. Oral antimuscarinic agents have been widely used as a first-line treatment option for NDO. However, this class of medications is ineffective in some patients and may also cause adverse effects (AEs), such as dry mouth, constipation, or blurred vision [3]. Oxybutynin is the antimuscarinic agent most used in detrusor overactivity [6,7] and represents the gold standard to which other drugs are compared. The acceptance of oxybutynin over a long period of time is low, however: $91 \%$ at 3 months, which decreases to $18 \%$ after 1 year [8].

The use of extended-release antimuscarinics such as oxybutynin, tolterodine, darifenacin, and solifenacin improves the acceptance by patients owing to a decrease in side effects [9]. The antimuscaric agents promote an increase in bladder capacity and compliance and a decrease of detrusor involuntary contraction.

Intradetrusor injections of botulinum toxin A (BoTN-A) have been used as a second-line option for patients who are unable
Corresponding author: Carlos Arturo Levi D’Ancona

Division of Urology, University of Campinas School of Medicine, Rua Dr.

Miguel Penteado, 1073 Campinas, Sao Paulo, CEP: 13070-118, Brazil

Tel: +55-19-32422488 / Fax: +55-19-32422488 / E-mail: cdancona@uol.com.br

Submitted: September 7, 2012 / Accepted after revision: September 17, 2012
This is an Open Access article distributed under the terms of the Creative Commons Attribution Non-Commercial License (http://creativecommons.org/licenses/by-nc/3.0/) which permits unrestricted non-commercial use, distribution, and reproduction in any medium, provided the original work is properly cited. 
to tolerate antimuscarinic drugs or for those whose clinical response to this treatment is unsatisfactory [10].

\section{Mechanism of Action}

Botulinum toxin is produced by Clostridium botulinum and is regarded as the most potent biological poison known to man. Seven immunologically distinct neurotoxins are designated $\mathrm{A}$ to $\mathrm{G}$, and to date only BoTN-A in BoTN-B are in clinical use. Some BoTN-A is commercially available and the most known are onabotulinumtoxin (Allergan, Westport, Ireland), abobotulinumtoxin (Ipsen Ltd., Berkshire, UK), and incobotulinumtoxin (Merz Pharmaceuticals, Frankfurt am Main, Germany). There are similarities between the products; however, they have different doses, efficacy, and safety profiles. One needs to keep in mind that the different preparations are not comparable. Lethal Dose 50 units are not equivalent because manufacturers use different methods of purification, formulation, and unit determination.

BoTN-A exerts paralyzing effects by inhibiting acetylcholine release from the motor nerve into the neuromuscular junction with inhibitory effects on autonomic and somatic neurotransmission. After intramuscular injection of BoTN-A, a temporary chemodenervation and relaxation of skeletal and smooth muscles can be achieved.

The effects of BoTN-A on the neuromuscular junctions have been extensively described. BoTN-A promotes the inhibition acetylcholine release, which results in muscle relaxation [11]. There is also evidence that BoTN-A inhibits other neurotransmitters, such as adenosine triphosphate, and neuropeptides, such as substance P [12]. It has also been suggested that BoTNA might play a role in regulating the expression of purinergic receptors and capsaicin in the afferent neurons of the bladder wall [13].

The first report of direct BoTN-A injection into the detrusor muscle was published in 2000 by Schurch et al. [14], who studied patients with DO secondary to SCI. Since that study, BoTNA has proven effective in improving urodynamic parameters, such as maximum cystometric capacity (MCC), maximum detrusor pressure (Pdet $\mathrm{Pax}_{\mathrm{x}}$ ), and bladder compliance. It also has a positive impact on QoL [11]. Recently, Wu et al. [15] reported that the cost-benefit ratio of BoTN-A treatment was superior to that of antimuscarinic drugs for refractory urge urinary incontinence. BoTN-A has also been shown to be associated with a significant reduction in the cost of drugs for urinary tract infections [16].
In the past, the objective of the treatment of patients with NDO was to keep the patients alive and decrease related complications. With improvements in care, better treatment, and longer life expectancies, QoL becomes a very important aspect of treatment. Many questionnaires have been developed to assess QoL, and both a general and a specific QoL questionnaire are necessary to evaluate patients with NDO. The Qualiveen questionnaire was developed in France specifically for neurogenic patients and has questions to evaluate general and specific domains. This questionnaire was translated and validated in the Portuguese language.

The purposes of this study were to verify the results of intradetrusor injection of BoTN-A on urodynamic parameters and the QoL of patients with NDO.

\section{MATERIALS AND METHODS}

This clinical trial was approved by the Research Ethical Committee at University of Campinas, and an informed consent form was read to each patient and signed before study enrollment.

A total of 39 patients with SCI and NDO were recruited for this study. Five were excluded because they did not fulfill the criteria or did not agree to participate in the study. The inclusion criteria were defined as male and female patients over 18 years of age who had had SCI for at least 12 months and who had been regularly undergoing clean intermittent self-catheterization (CISC). Exclusion criteria were pregnancy, the desire to become pregnant during the study period, breastfeeding, the use of anticoagulants or a report of a blood coagulation disorder, neuromuscular transmission disorder, the use of any intravesical pharmacologic agents, or previous use of BoTN-A. Patients discontinued the use of any antimuscarinic medication 7 days before the baseline evaluation.

All 34 patients were clinically evaluated in accordance with the neurologic scale developed by the American Spinal Injury Association [17]. Laboratory evaluation included measurement of serum urea and creatinine, urinalysis, and urine culture with antimicrobial susceptibility testing and imaging as appropriate.

Urinary continence was defined as an absence of urinary leakage in the intervals between CISCs [18]. Patients were required to perform a 3-day bladder diary, reporting the number and times of CISC, volume of each CISC, and incontinence episodes between them. All patients completed the Qualiveen questionnaire [19]. 
Urodynamic study was performed in accordance with the recommendations set forth by the International Continence Society [20]. Urodynamic parameters were defined according to the International Continence Society standardization [21].

The bladder diary and QoL evaluations were performed at the beginning of the study and were repeated at weeks 4 and 24 . Patients reported AEs as applicable during the follow-up visits.

Patients were treated with intradetrusor injections of $300 \mathrm{U}$ onabotulinumtoxin-A (BTX-A; BotoX, Allergan). The urodynamic parameters were measured at the beginning and at the end of the study ( 24 weeks). QoL was evaluated by the

Table 1. Demographic data $(n=28)$

\begin{tabular}{lc}
\hline Variable & Value \\
\hline Gender & $23 / 5$ \\
Male/female & \\
Age (yr) & $33 \pm 11(19-61)$ \\
Mean \pm SD (range) & \\
Time of injury (mo) & $23 \pm 8(12-47)$ \\
Mean \pm SD (range) & \\
Neurological injury level & 21 \\
T1-T6 & 7 \\
T7- T12 & 0 \\
L1 & \\
ASIA scores & 16 \\
A & 10 \\
B & 2 \\
C & 0 \\
D &
\end{tabular}

ASIA, American Spinal Injury Association.
Qualiveen questionnaire.

Patients were administered antibiotics for 7 days on the basis of urine culture results. Injections were performed on the third day of antibiotic use in a surgical setting, with the patient under intravenous anaesthesia with $2 \mathrm{mg} / \mathrm{kg}$ propofol. Three hundred units of BTX-A were applied, as previously described by Schurch et al. [14].

\section{Statistical Analysis}

This study was planned with an error of $4 \%$ and a power of $80 \%$. The Wilcoxon test was used to compare intragroup variations in numeric variables between the evaluations conducted at baseline and at the end of the study. The significance level adopted for the statistical tests was 5\%. We used SAS ver. 9.1.3 (SAS Institute Inc., Cary, NC, USA) for all analyses.

\section{RESULTS}

Six patients did not complete the study. Of the 28 patients who did, $80.3 \%$ were male. The demographic data of these patients are summarized in Table 1.

BTX-A treatment resulted in a significant increase in MCC and bladder compliance as well as a reduction in Pdet max $_{\max }$ and in the number of episodes of incontinence per 24 hours. Analysis of the three QoL assessment answers showed a strongly positive effect of BTX-A on QoL, as shown in Table 2.

Four patients (14\%) presented no changes in urodynamic parameters or QoL scores. Furthermore, one of these patients actually suffered from a reduction in bladder compliance. Macroscopic hematuria was present during the first 24 hours in eight patients (28\%) treated with BTX-A. No systemic AEs

Table 2. Comparison of number of incontinence episodes per 24 hours, urodynamic parameters, and QoL scores at baseline and after 24 weeks

\begin{tabular}{|c|c|c|c|}
\hline Parameter & Baseline & 24 wk & P-value ${ }^{a)}$ \\
\hline No. of incontinence episodes $/ 24 \mathrm{hr}$ & $7 \pm 1(6-9)$ & $1 \pm 3(0-11)$ & $<0.001$ \\
\hline $\operatorname{MCC}(\mathrm{mL})$ & $172 \pm 33(115-225)$ & $461 \pm 139(130-600)$ & $<0.001$ \\
\hline $\operatorname{Pdet}_{\max }\left(\mathrm{cmH}_{2} \mathrm{O}\right)$ & $79 \pm 21(36-114)$ & $30 \pm 27(5-105)$ & $<0.001$ \\
\hline Bladder compliance $\left(\mathrm{mL} / \mathrm{cmH}_{2} \mathrm{O}\right)$ & $15 \pm 3(6-20)$ & $40 \pm 24(13-120)$ & $<0.001$ \\
\hline Qualiveen, SIUP & $3.38 \pm 0.39(2.45-4.00)$ & $1.90 \pm 0.71(1.15-4.00)$ & $<0.001$ \\
\hline Qualiveen, QoL index & $-1.28 \pm 0.45(-2.00--0.33)$ & $-0.65 \pm 0.49(-1.56-0.11)$ & $<0.001$ \\
\hline
\end{tabular}

Values are presented as mean $\pm \mathrm{SD}$ (range).

MCC, maximum cystometric capacity; Pdet ${ }_{\max }$, maximum detrusor pressure; QoL, quality of life; SIUP, specific impact of urinary problems.

${ }^{a)}$ Wilcoxon test. 
were reported.

\section{DISCUSSION}

Patients treated with BTX-A demonstrated significant improvement in all urodynamic parameters evaluated. In agreement with the findings of the present study, Reitz et al. [22] reported a significant increase in mean MCC and bladder compliance after 12 weeks. That same report also showed a significant decrease in Pdet max.

In the present study, we observed a significant reduction in the number of episodes of urinary incontinence per 24 hours in the BTX-A-treated patients. Similar results were reported by Giannantoni et al. [23], who treated 17 SCI patients with Botox and followed their progress for more than 6 years. That study demonstrated a significant reduction in the mean number of episodes of incontinence per day after a 1-year follow-up.

BTX-A treatment resulted in a significantly positive impact on the QoL of patients. Schurch et al. [14] reported an improvement in the QoL of 59 patients allocated into three groups and treated with either 300 U BoTN-A, 200 U BoTN-A, or placebo and followed for 24 weeks. That study demonstrated a significant improvement in the QoL scores of the BoTN-A-treated patients.

The improvement in urodynamic parameters resulted in an extremely positive effect on the QoL. According to Pannek and Kullik [1], treatment regimens that result in improved urodynamic outcome and urinary continence are associated with an improvement in QoL. It is our belief that another factor that contributed to the improvement of the QoL in the present study was the superior tolerability profile of BTX-A, because no AEs were reported in these patients.

Urinary continence rates were significantly higher (60\%). Karsenty et al. [24] reported a urinary continence rate ranging from 40 to $80 \%$.

Mild macroscopic hematuria in the first 24 hours after injection was found in eight patients (28\%) who received BTX-A treatments. Karsenty et al. [24] reported an incidence of mild hematuria of 2 to $21 \%$.

No systemic AEs following BTX-A treatment were observed in the present study. The injection technique used was the same as that described by Schurch et al. [25]. Karsenty et al. [24] reported that muscular weakness may be related to factors such as the BTX-A dose, the interval between applications, or the injection technique, as a consequence of detrusor perforation during injection and diffusion of the drug into neighboring structures. In four patients treated with BTX-A (14\%), no changes were found in the urodynamic parameters or in the QoL scores, and one patient actually presented a reduction in bladder compliance. Reitz et al. [22] identified 9 patients out of a total of 200 (4.5\%) who failed to respond to BTX-A treatment and who, as in the present study, experienced no clinical or urodynamic benefits. The reasons for the lack of response in these patients could not be determined. However, the possibility of an error occurring during dilution or injection of BTX-A cannot be excluded. According to Compérat et al. [26], BTX-A injections do not lead to any increase in fibrotic activity inside the bladder wall.

From an economic point of view, Wefer et al. [16] recently reported that BTX-A treatment results in a significant reduction in the morbidity associated with NDO as well as in the costs associated with medications necessary to treat urinary tract infections and devices for incontinence.

In conclusion, comparing both the objective (urodynamic study) and subjective (QoL questionnaire) data, BTX-A was found to be effective for all parameters evaluated.

\section{CONFLICT OF INTEREST}

No potential conflict of interest relevant to this article was reported.

\section{REFERENCES}

1. Pannek J, Kullik B. Does optimizing bladder management equal optimizing quality of life? Correlation between health-related quality of life and urodynamic parameters in patients with spinal cord lesions. Urology 2009;74:263-6.

2. Grise P, Ruffion A, Denys P, Egon G, Chartier Kastler E. Efficacy and tolerability of botulinum toxin type $\mathrm{A}$ in patients with neurogenic detrusor overactivity and without concomitant anticholinergic therapy: comparison of two doses. Eur Urol 2010;58:759-66.

3. West DA, Cummings JM, Longo WE, Virgo KS, Johnson FE, Parra RO. Role of chronic catheterization in the development of bladder cancer in patients with spinal cord injury. Urology 1999;53:292-7.

4. Lapides J, Diokno AC, Silber SJ, Lowe BS. Clean, intermittent selfcatheterization in the treatment of urinary tract disease. J Urol 1972; 107:458-61.

5. Feifer A, Corcos J. Contemporary role of suprapubic cystostomy in treatment of neuropathic bladder dysfunction in spinal cord in- 
jured patients. Neurourol Urodyn 2008;27:475-9.

6. Karsenty G, Denys P, Amarenco G, De Seze M, Game X, Haab F, et al. Botulinum toxin A (Botox) intradetrusor injections in adults with neurogenic detrusor overactivity/neurogenic overactive bladder: a systematic literature review. Eur Urol 2008;53:275-87.

7. Lee YS, Choo MS, Lee JY, Oh SJ, Lee KS. Symptom change after discontinuation of successful antimuscarinic treatment in patients with overactive bladder symptoms: a randomised, multicentre trial. Int J Clin Pract 2011;65:997-1004.

8. Kay GG, Granville LJ. Antimuscarinic agents: implications and concerns in the management of overactive bladder in the elderly. Clin Ther 2005;27:127-38.

9. Chapple CR. Muscarinic receptor antagonists in the treatment of overactive bladder. Urology 2000;55(5A Suppl):33-46.

10. Di Stasi SM, Giannantoni A, Vespasiani G, Navarra P, Capelli G, Massoud R, et al. Intravesical electromotive administration of oxybutynin in patients with detrusor hyperreflexia unresponsive to standard anticholinergic regimens. J Urol 2001;165:491-8.

11. Abdel-Meguid TA. Botulinum toxin-A injections into neurogenic overactive bladder--to include or exclude the trigone? A prospective, randomized, controlled trial. J Urol 2010;184:2423-8.

12. Schurch B, de Seze M, Denys P, Chartier-Kastler E, Haab F, Everaert $\mathrm{K}$, et al. Botulinum toxin type a is a safe and effective treatment for neurogenic urinary incontinence: results of a single treatment, randomized, placebo controlled 6-month study. J Urol 2005;174: 196-200.

13. Apostolidis A, Haferkamp A, Aoki KR. Understanding the Role of Botulinum Toxin A in the Treatment of the Overactive BladderMore than Just Muscle Relaxation. Eur Urol Suppl 2006;5:670-8.

14. Schurch B, Stohrer M, Kramer G, Schmid DM, Gaul G, Hauri D. Botulinum-A toxin for treating detrusor hyperreflexia in spinal cord injured patients: a new alternative to anticholinergic drugs? Preliminary results. J Urol 2000;164(3 Pt 1):692-7.

15. Wu JM, Siddiqui NY, Amundsen CL, Myers ER, Havrilesky LJ, Visco AG. Cost-effectiveness of botulinum toxin a versus anticholinergic medications for idiopathic urge incontinence. J Urol 2009; 181:2181-6.

16. Wefer B, Ehlken B, Bremer J, Burgdorfer H, Domurath B, Hampel $\mathrm{C}$, et al. Treatment outcomes and resource use of patients with neurogenic detrusor overactivity receiving botulinum toxin A (BOTOX) therapy in Germany. World J Urol 2010;28:385-90.
17. Maynard FM Jr, Bracken MB, Creasey G, Ditunno JF Jr, Donovan WH, Ducker TB, et al. International Standards for Neurological and Functional Classification of Spinal Cord Injury. American Spinal Injury Association. Spinal Cord 1997;35:266-74.

18. Pannek J, Gocking K, Bersch U. Long-term effects of repeated intradetrusor botulinum neurotoxin A injections on detrusor function in patients with neurogenic bladder dysfunction. BJU Int 2009; 104:1246-50.

19. D’Ancona CA, Tamanini JT, Botega N, Lavoura N, Ferreira R, Leitao $\mathrm{V}$, et al. Quality of life of neurogenic patients: translation and validation of the Portuguese version of Qualiveen. Int Urol Nephrol 2009;41:29-33.

20. Schafer W, Abrams P, Liao L, Mattiasson A, Pesce F, Spangberg A, et al. Good urodynamic practices: uroflowmetry, filling cystometry, and pressure-flow studies. Neurourol Urodyn 2002;21:261-74.

21. Abrams P, Cardozo L, Fall M, Griffiths D, Rosier P, Ulmsten U, et al. The standardisation of terminology in lower urinary tract function: report from the standardisation sub-committee of the International Continence Society. Urology 2003;61:37-49.

22. Reitz A, Stohrer M, Kramer G, Del Popolo G, Chartier-Kastler E, Pannek J, et al. European experience of 200 cases treated with botulinum-A toxin injections into the detrusor muscle for urinary incontinence due to neurogenic detrusor overactivity. Eur Urol 2004; 45:510-5.

23. Giannantoni A, Mearini E, Del Zingaro M, Porena M. Six-year follow-up of botulinum toxin A intradetrusorial injections in patients with refractory neurogenic detrusor overactivity: clinical and urodynamic results. Eur Urol 2009;55:705-11.

24. Karsenty G, Reitz A, Lindemann G, Boy S, Schurch B. Persistence of therapeutic effect after repeated injections of botulinum toxin type A to treat incontinence due to neurogenic detrusor overactivity. Urology 2006;68:1193-7.

25. Schurch B, Denys P, Kozma CM, Reese PR, Slaton T, Barron RL. Botulinum toxin A improves the quality of life of patients with neurogenic urinary incontinence. Eur Urol 2007;52:850-8.

26. Compérat E, Reitz A, Delcourt A, Capron F, Denys P, ChartierKastler E. Histologic features in the urinary bladder wall affected from neurogenic overactivity: a comparison of inflammation, oedema and fibrosis with and without injection of botulinum toxin type A. Eur Urol 2006;50:1058-64. 